\title{
Rastreamento da Sindrome de Down com Uso de Escore de Múltiplos Parâmetros Ultra-sonográficos
}

\author{
Ultrasound Screening for Down Syndrome Using a Multiparameter Score
}

\author{
Victor Bunduki, Rodrigo Ruano, Adolfo Wenjaw Liao \\ Claudia Chiba, Seizo Miyadahira, Marcelo Zugaib
}

\section{RESUMO}

Objetivos: calcular a sensibilidade, especificidade e posteriormente os valores preditivos positivo e negativo dos escores ultra-sonográficos na síndrome de Down.

Pacientes e Métodos: a sensibilidade e especificidade dos sinais ultra-sonográficos para a sindrome de Down foram calculadas por meio de escores em um estudo prospectivo realizado em população de alto risco para aneuploidia, entre a $16^{a}$ e $24^{a}$ semanas de gestação, que se mostrou desfavorável aos procedimentos invasivos após aconselhamento genético. Os sinais e os valores para a confecção dos escores foram: relação do comprimento do fêmur/pé <0,9 (1), espessura nucal $>5 \mathrm{~mm}$ (2), diâmetro pielocalicial $\geq 5 \mathrm{~mm}$ (1), ossos próprios do nariz $<6 \mathrm{~mm}$ (1), ausência ou hipoplasia da falange média do $5^{\circ}$ dedo (1) e alterações estruturais maiores (2). Seguimento completo foi obtido caso a caso. A amniocentese genética foi proposta nos casos de escores 2 ou mais.

Resultados: 963 pacientes foram submetidas a exames ultra-sonográficos no periodo de outubro de 93 a dezembro de 97. A idade gestacional média foi de 19,6 semanas (16 a 24 semanas). A idade materna variou de 35 a 47 anos (média de 38,8) e 18 casos (1,87\%) de síndrome de Down foram observados. A sensibilidade foi de 94,5\% para escores 1 e $73 \%$ para escores 2 (taxa de falso-positivos de 9,8\% para escores 1 e de 4,1\% para escores 2). A sensibilidade e especificidade individual para cada sinal foram: comprimento fêmur $/$ pé = $16,7 \%$ (3/18) e 96,8\% (915/945); ossos próprios do nariz $=22,2 \%(4 / 18)$ e 92,1\% (870/945); espessamento nucal = 44,4\% (8/18) e 96,5\% (912/945); diâmetro pielocalicial = $38,9 \%$ (7/18) e 94,3\% (891/945); ausência da falange média do $5^{\circ}$ dedo $=22,2 \%(4 / 18) e$ 98,5\% (912/945); malformações = 22,2\% (4/18) e 98,2\% (928/945).

Setor de Medicina Fetal da Clínica Obstétrica do Hospital das

Clínicas da Faculdade de Medicina da Universidade de

São Paulo

Correspondência:

Dr. Victor Bunduki

Rua Dr. Enéas de Carvalho Aguiar, 255 - $10^{\circ}$ andar

Cerqueira César - 05403-000 - São Paulo - SP 
Conclusões: a sensibilidade geral para escore 1 foi bastante alta, porém taxas de falso-positivos também oforam. Para escore 2, a sensibilidade foi ainda boa (73\%), com taxa de falso-positivos aceitáveis (4,1\%). Os valores preditivos positivo e negativo podem ser calculados conforme a prevalência de cada situação (diferentes idades maternas). Mais casos são necessários para concluir sobre este método de rastreamento (especialmente na população de baixo risco), apesar de este sistema ter se mostrado útil para pacientes de alto risco que não optam pelo diagnóstico invasivo.

PALAVRAS-CHAVE: Síndrome de Down. Ultra-sonografia. Diagnóstico pré-natal. Idade materna. Amniocentese.

Introdução

Em países desenvolvidos, o diagnóstico citogenético pré-natal para gestantes consideradas de risco para aneuploidias fetais está muito difundido $^{12}$. A principal indicação para essa propedêutica invasiva (amniocentese e biópsia de vilo corial) é a idade materna avançada. A probabilidade de anomalia cromossômica ao nascimento é de aproximadamente $0,5 \%$ na mulher de 35 anos, atingindo 1,2\% naquela de 37 anos, $6 \%$ na mulher de 43 anos e finalmente atinge taxas de $11 \%$ na mulher de 45 anos. A sindrome de Down é responsável por mais ou menos a metade dos casos de aneuploidia ${ }^{4,5,6}$.

No Brasil, apesar da divulgação, esse recurso é pouco utilizado na prática clínica. Em nossa experiência, $62 \%$ das mulheres com idade igual ou superior a 35 anos não optam pela propedêutica invasiva mesmo após aconselhamento genético. Além disso, a maioria das gestantes inicia o prénatal no segundo trimestre e realiza o seu primeiro exame ultra-sonográfico obstétrico nesse período. Com isso, no Brasil o acesso ao diagnóstico invasivo torna-se muito limitado ${ }^{5}$.

Ao considerar, a grosso modo, a taxa de perda fetal associada ao procedimento invasivo diagnóstico como sendo de aproximadamente $1 \%$, estabeleceu-se que mulheres com menos de 35 anos não se beneficiam verdadeiramente desse recurso, em razão do maior número de perdas de fetos normais do que identificação de doentes. Porém, a não-realização rotineira do diagnóstico invasivo faz com que uma percentagem significativa (cerca de 70\%) dos fetos com aneuploidia nasça de pacientes com idade inferior a 35 anos, em especial a faixa etária entre os 30 e 34 anos de idade na qual as aberrações citogenéticas têm prevalência considerável e dentro da qual as pacientes não têm indicação de propedêutica invasiva ${ }^{5,13}$.
Nesse sentido, outros métodos de rastreamento das aneuploidias assumem importância, na medida em que podem determinar o aumento do risco de uma anomalia cromossômica em pacientes que previamente não apresentavam indicação para o estudo citogenético. Podem também determinar a redução do risco em gestantes com idade igual ou superior a 35 anos que não teriam acesso ou não optariam pelo estudo invasivo.

Com isso o objetivo desse estudo foi calcular a sensibilidade, especificidade e os valores preditivos positivo e negativo de determinados sinais e escores ultra-sonográficos do segundo trimestre relacionados ao diagnóstico de síndrome de Down, para posterior utilização na prática clínica.

\section{Pacientes e Métodos}

No período de outubro de 1993 a dezembro de 1997, foram acompanhadas, no Setor de Medicina Fetal da Clínica Obstétrica da Faculdade de Medicina da Universidade de São Paulo, pacientes com idade igual ou superior a 35 anos e que não optaram pelo diagnóstico invasivo após consulta de aconselhamento genético. O estudo foi realizado de maneira prospectiva.

Foram avaliados sinais ultra-sonográficos em idade gestacional entre 16 e 24 semanas completas, que receberam notas iguais a 1 ou 2 . Os parâmetros com valor 1 para o escore final foram: 1) relação entre o comprimento do fêmur/pé < 0,9. O fêmur foi medido segundo técnica habitual, em corte longitudinal com o osso perpendicular ao eixo de ondas. O comprimento do pé foi obtido em corte coronal da planta do pé e medido do talus ao artelho mais longo; 2) diâmetro pielocalicial $\geq 5 \mathrm{~mm}$, obtido medindo-se a pelve, em corte transversal do rim, 
no sentido ântero-posterior; 3) ossos próprios do nariz $<6 \mathrm{~mm}$, medidos em corte sagital mediano da face, da base à ponta do nariz, e 4) ausência ou hipoplasia da falange média do 5 엉 dedo. Dois outros parâmetros ultra-sonográficos receberam nota 2: 5) espessura da nuca, medida da pele ao osso occipital em corte transversal passando pelo cerebelo e fossa posterior, e 6) presença de alterações estruturais, como por exemplo anomalias cardíacas, dilatação ventricular, ascite, pé torto, etc. Dessa maneira, o escore mínimo era igual a 0 (zero) e o máximo de 8.

Assim, a especificidade e sensibilidade para a sindrome de Down foram calculadas para os diferentes valores dos escores ultra-sonográficos.

Foi considerado apenas 1 exame por paciente no intervalo de idade gestacional determinado acima. Os exames ultra-sonográficos foram realizados inicialmente pelo primeiro autor e, posteriormente, por mais 3 médicos operadores, todos treinados para a obtenção dos cortes necessários. Em nenhuma paciente o operador conhecia o resultado do cariótipo fetal. Somente foram incluídos os exames nos quais todos os parâmetros propostos foram obtidos.

O seguimento completo quanto à normalidade ou à presença de aneuploidia no produto da concepção foi realizado caso a caso ao nascimento ou após exame citogenético quando a paciente optou em segunda ordem pela realização do estudo do líquido amniótico, ou ainda, a posteriori, por meio do recebimento de cartas respondidas pelas pacientes ou por contato telefônico. Para fins assistenciais, a amniocentese diagnóstica para o estudo citogenético foi proposta ao casal nas situações em que estavam presentes escores de 2 ou mais.

\section{Resultados}

Um total de 963 exames ultra-sonográficos foram realizados no período de outubro de 1993 a dezembro de 1997. A idade materna variou de 35 a 47 anos, com média de 38,8 , e a idade gestacional variou de $16^{\mathrm{a}}$ a $24^{\mathrm{a}}$ semanas de gestação (média de 19,6). Ocorreram 18 casos de fetos ou recémnascidos com o diagnóstico de síndrome de Down correspondendo a uma prevalência de 1,87\% .

A Tabela 1 apresenta a sensibilidade e a especificidade para sindrome de Down escore igual ou superior a 1 , que foram respectivamente de $94,5 \%(17 / 18)$ e $90,2 \%(852 / 945)$. A taxa de falsopositivos observada para o mesmo escore foi $9,8 \%$. A Tabela 2 apresenta a sensibilidade e especificidade do escore igual ou maior que 2 , que foram, respectivamente, $73 \%(13 / 18)$ e $95,9 \%$ (908/945), com uma taxa de falso-positivos de 4,1\%.

Tabela 1 - Sensibilidade e especificidade geral da presença de sinais ultra-sonográficos para Síndrome de Down cujo escore é igual ou maior que 1.

\begin{tabular}{lccr}
\hline & Sinais presentes & Sinais ausentes & Total \\
\hline Síndrome de Down & 17 & 1 & 18 \\
Controle & 93 & 852 & 945 \\
Total & $\mathbf{1 1 0}$ & $\mathbf{8 5 3}$ & $\mathbf{9 6 3}$ \\
\hline
\end{tabular}

Sensibilidade: $94,5 \%$

Especificidade: $90,2 \%$

Falso-positivos: 9,8\%

Tabela 2 - Sensibilidade e especificidade geral da presença de sinais ultra-sonográficos para Síndrome de Down com escore igual a 2 (2 ou mais sinais presentes).

\begin{tabular}{lccr}
\hline & Sinais presentes & Sinais ausentes & Total \\
\hline Síndrome de Down & 13 & 5 & 18 \\
Controle & 37 & 908 & 945 \\
Total & $\mathbf{5 0}$ & $\mathbf{9 1 3}$ & $\mathbf{9 6 3}$ \\
\hline
\end{tabular}

Sensibilidade: $73 \%$

Especificidade: $95,9 \%$

Falso-positivos: 4,1\%

A sensibilidade da presença isolada da relação do comprimento fêmur/pé $<0,9$ foi de $16,7 \%$ (3/18) (Tabela 3), com especificidade de $96,8 \%(915 / 945)$ e taxa de falso-positivos igual a $3,2 \%$. Em relação ao comprimento dos ossos próprios do nariz, observaram-se sensibilidade de $22,2 \%$ (4/18), especificidade de 92,1\% (870/945) e 7,9\% de falso-positivos (Tabela 4). A medida isolada do diâmetro pielocalicial $\geq 5 \mathrm{~mm}$ (Tabela 5) apresentou sensibilidade de 38,9\% (7/18), especificidade de $94,3 \%$ (891/945) e taxa de falsopositivos de 5,7\%. Avaliando-se, isoladamente, o sinal ultra-sonográfico da ausência da falange média do 5o dedo (Tabela 6), foi observado sensibilidade de $22,2 \%$ (4/18), especificidade de $96,5 \%(912 / 945)$ e $1,5 \%$ de falso-positivos.

A Tabela 7 demonstra o valor da medida da espessura da nuca como dado isolado no rastreamento da sindrome de Down, apresentando sensibilidade de 44,4\% (8/18), especificidade de $96,5 \%(912 / 945)$ e taxa de falso-positivos igual a $3,5 \%$. O valor da presença de outras malformações detectadas no exame ultra-sonográfico do segundo trimestre como dado isolado no rastreamento da 
Síndrome de Down está representado na Tabela 8, isto é, sensibilidade de $22,2 \%$ (4/18), especificidade de $98,2 \%(928 / 945)$ e taxa de falso-positivos igual a $1,8 \%$. As malformações presentes foram malformação cardíaca $(n=2)$, pé torto $(n=1)$ e dilatação de ventrículos cerebrais $(n=1)$.

Tabela 3 - Sensibilidade e especificidade da relação do comprimento fêmur/pé $<0,9$ para o diagnóstico da Síndrome de Down.

\begin{tabular}{lccr}
\hline & Sinal presente & Sinal ausente & Total \\
\hline Síndrome de Down & 3 & 15 & 18 \\
Controle & 30 & 915 & 945 \\
Total & $\mathbf{3 3}$ & $\mathbf{9 3 0}$ & $\mathbf{9 6 3}$ \\
\hline
\end{tabular}

Sensibilidade: $16,7 \%$

Especificidade: $96,8 \%$

Falso-positivos: $3,2 \%$

Tabela 4 - Sensibilidade e especificidade do comprimento dos ossos próprios do nariz $<6 \mathrm{~mm}$ para diagnóstico da Síndrome de Down.

$$
\text { Sinal presente Sinal ausente Total }
$$

\begin{tabular}{lrrr}
\hline Síndrome de Down & 4 & 14 & 18 \\
Controle & 75 & 870 & 945 \\
Total & $\mathbf{7 9}$ & $\mathbf{8 8 4}$ & $\mathbf{9 6 3}$ \\
\hline
\end{tabular}

Sensibilidade: $22,2 \%$

Especificidade: $92,1 \%$

Falso-positivos: $7,9 \%$

Tabela 5 - Sensibilidade e especificidade do diâmetro pielocalicial $\geq 5 \mathrm{~mm}$ para 0 diagnóstico da Síndrome de Down.

\begin{tabular}{lccr}
\hline & Sinal presente & Sinal ausente & Total \\
\hline Síndrome de Down & 7 & 11 & 18 \\
Controle & 54 & 891 & 945 \\
Total & $\mathbf{6 1}$ & $\mathbf{9 0 2}$ & $\mathbf{9 6 3}$ \\
\hline
\end{tabular}

Sensibilidade: $38,9 \%$

Especificidade: $94,3 \%$

Falso-positivos: $5,7 \%$

Tabela 6 - Sensibilidade e especificidade da ausência da falange média do $5^{0}$ dedo para o diagnóstico da Síndrome de Down.

$$
\text { Sinal presente Sinal ausente Total }
$$

\begin{tabular}{lrrr}
\hline Síndrome de Down & 4 & 14 & 18 \\
Controle & 33 & 912 & 945 \\
Total & $\mathbf{3 7}$ & $\mathbf{9 2 6}$ & $\mathbf{9 6 3}$ \\
\hline
\end{tabular}

Sensibilidade: $22,2 \%$

Especificidade: $96,5 \%$

Falso-positivos: $1,5 \%$
Tabela 7 - Sensibilidade e especificidade da espessura nucal para o diagnóstico da Síndrome de Down.

\begin{tabular}{lccr}
\hline & Sinal presente & Sinal ausente & Total \\
& & 10 & 18 \\
Síndrome de Down & 8 & 912 & 945 \\
Controle & 33 & $\mathbf{9 2 2}$ & $\mathbf{9 6 3}$ \\
Total & $\mathbf{4 1}$ & & \\
\hline
\end{tabular}

Sensibilidade: $44,4 \%$

Especificidade: $96,5 \%$

Falso-positivos: 3,5\%

Tabela 8 - Sensibilidade e especificidade da presença de alterações estruturais fetais para o diagnóstico da Síndrome de Down.

\begin{tabular}{lccr} 
& Sinal presente & Sinal ausente & Total \\
\hline Síndrome de Down & 4 & 14 & 18 \\
Controle & 17 & 928 & 945 \\
Total & $\mathbf{2 1}$ & $\mathbf{9 4 2}$ & $\mathbf{9 6 3}$ \\
\hline
\end{tabular}

Sensibilidade: $22,2 \%$

Especificidade: $98,2 \%$

Falso-positivos: $1,8 \%$

\section{Discussão}

O diagnóstico das anomalias cromossômicas fetais no segundo trimestre pode ser realizado por meio da propedêutica invasiva (basicamente pela amniocentese para cariótipo) $)^{8,9}$. Por outro lado, o rastreamento para cálculo de risco destas anormalidades pode ser efetuado por meio da ultrasonografia ${ }^{3}$. O exame ultra-sonográfico pode identificar sinais que geralmente estão presentes nas aneuploidias, mas que podem também ser observados em alguns fetos normais ${ }^{1,3}$.

Com isso, o método de rastreamento considerado ideal é aquele que apresenta elevada sensibilidade para não deixar de identificar casos dessa doença, além de ter especificidade alta para reduzir o índice de falso-positivos. Já o valor preditivo positivo (risco real informado à paciente) dependerá estritamente da taxa de prevalência da doença na população. No caso das anomalias cromossômicas fetais, os valores preditivos (riscos) variam na dependência direta da idade da mulher e da idade gestacional.

Para que tenha utilidade clínica, todo rastreamento requer o conhecimento de sua sensibilidade, especificidade e a distribuição dos 
valores preditivos positivos e negativos em relação às diversas faixas de prevalência da doença na população estudada.

Em medicina fetal, outro fato importante é que o resultado positivo de um teste de rastreamento normalmente indica um procedimento diagnóstico que é invasivo para o feto e traz, conseqüentemente, um risco de abortamento. Nesse sentido, a aplicabilidade do método adquire importância ainda maior, especialmente em relação à taxa de falso-positivos.

Ao considerar somente a idade materna avançada como indicador para rastreamento, observa-se sensibilidade em torno de 30\% (visto que cerca de $70 \%$ das sindromes de Down ocorrem em mulheres com menos de 35 anos) e as taxas de falso-positivos variam de 199/200 (mulheres de 35 anos) até 89/100 (mulheres de 45 anos). Em média são necessários 140 estudos citogenéticos invasivos para diagnosticar 1 feto com trissomia do 21 (mulheres acima de 35 anos e com média de 37 anos) ${ }^{4}$. Após a introdução do rastreamento bioquímico materno, essa taxa aumentou para 1 caso positivo para 60 a 90 procedimentos invasivos $^{1,14}$.

Em nosso meio, onde o diagnóstico pré-natal não adquiriu estratégias bem definidas de oferta à população, sabemos que entre as mulheres de idade avançada, às quais é oferecido aconselhamento genético, apenas $32 \%$ opta pela diagnóstico invasivo $^{2}$.

Assim, número significativo destas mulheres opta ou por não fazer nada ou pelo rastreamento iniciado no primeiro trimestre com a metodologia da translucência nucal. O programa da translucência nucal tem $77 \%$ de sensibilidade para uma taxa de falso-positivos de 5\% e nivel de corte de 1/300 (que é mais ou menos a prevalência da trissomia do 21 na mulher de 33 anos) ${ }^{10}$. A translucência nucal quando positiva indica $o$ caminho para a biópsia de vilo corial, que tem como principais limitações a taxa de abortamento (1\%) mais alta que a da amniocentese e a presença de resultados duvidosos que chegam até $2,5 \%$ (probabilidade de ter de repetir a coleta) ${ }^{7,10}$. Além do mais, os métodos de rastreamento não são excludentes. Seria de grande interesse, então, dispormos de outro método não-invasivo que pudesse auxiliar no rastreamento de aneuploidias.

Outro fator de considerável impacto sobre a utilidade do método de rastreamento do segundo trimestre é que muitas trissomias evoluem para abortamento até a $16^{\mathrm{a}}$ semana e mesmo mais tarde ao longo da gestação. Portanto, no rastreamento durante o primeiro trimestre podemos estar calculando riscos para uma prevalência artificialmente alta, pois muitos fetos anormais podem não passar da $14^{\mathrm{a}}$ ou $16^{\mathrm{a}}$ semana. Com isso, no segundo trimestre lidamos com prevalências mais baixas em relação ao primeiro. Estes dados são de fundamental importância e o conhecimento destes fatos influencia consideravelmente os valores preditivos tanto positivos quanto negativos dos testes de rastreamento ${ }^{5}$.

Porém, é interessante notar que a sensibilidade e a especificidade dizem somente respeito ao método de rastreamento estudado. Assim, de posse destes valores de sensibilidade e especificidade do método e conhecendo a prevalência da doença em uma faixa etária e em determinada idade gestacional, podemos calcular o risco real para a paciente, qualquer que seja a sua idade. Tanto o programa da translucência nucal quanto o teste bioquímico triplo de rastreamento nos fornecem este valor em fração, ao levar em conta estas informações. Portanto, nosso objetivo neste trabalho foi, primeiro, obter a sensibilidade e a especificidade de determinados sinais ultra-sonográficos em relação à síndrome de Down, para a posteriori podermos calcular os riscos reais em relação a cada idade. Estudamos pacientes com idade acima de 35 anos devido à maior prevalência da síndrome de Down nesta faixa etária o que permite obter mais facilmente estes valores.

A fim de exprimir a importância destes dados na prática clínica durante o pré-natal, podemos mostrar dois exemplos baseados na tabela de prevalência de síndrome de Down em relação à idade materna e à idade gestacional descrita por Snijders et al. ${ }^{11}$ e utilizando a sensibilidade e especificidade observadas em nosso estudo. Uma gestante de 33 anos (prevalência da síndrome de Down de 1/421 com 20 semanas), apresentando escore igual a 1 , tem um valor preditivo positivo de $2,3 \%$ (sensibilidade $=94,5 \%$, especificidade $=$ $90,2 \%)$, isto é, aumentando a chance de essa doença estar presente nessa gravidez ( 1 / 44 ao invés de 1/421). Por outro lado, para uma gestante de 36 anos (prevalência de sindrome de Down igual a $1 / 216$ com 20 semanas), apresentando escore igual a zero (sem nenhum sinal ultra-sonográfico), obtem-se valor preditivo negativo de 99,75\% (sensibilidade do escore $1=94,5 \%$ e especificidade do escore $1=90,2 \%$ ), isto é, a chance de síndrome de Down diminui de 1/216 para 1/4000. Se essa mesma paciente de 36 anos apresentar escore ultra-sonográfico igual a 1, ela passa a ter uma chance de o feto ser portador da síndrome de Down de $1 / 24$ (valor preditivo positivo $=4,4 \%$ ) ao invés dos $1 / 216$ iniciais.

Não existe, portanto, nenhum sinal que separe precisamente os individuos normais dos anormais, pois isto seria um sinal diagnóstico e 
não de triagem. Como não existe método ideal, deve-se considerar adequado aquele que se aproxima deste fato, ou seja, alta sensibilidade com taxas de falso-positivos (especificidade) aceitáveis.

Observamos alta sensibilidade da presença de escore 1 (94,5\%), porém com elevado índice de falso-positivos $(9,8 \%)$. Em relação à presença de escore 2 , a sensibilidade caiu (73\%), porém a especificidade aumentou consideravelmente $(95,9 \%)$.

Ao se considerar cada sinal ultra-sonográfico isolado, observamos, em nossa série, sensibilidade baixa com especificidade alta para sindrome de Down. A presença da prega nucal espessada foi o dado de maior sensibilidade (44\%), ao passo que ausência da falange média do quinto dedo foi o que apresentou maior especificidade $(98,5 \%)$.

Portanto, a identificação de um sinal isolado durante o exame ultra-sonográfico pode indicar a presença de anomalias citogenéticas, porém até $10 \%$ de fetos normais seriam triados se somente um dado fosse considerado. Assim, sugerimos a classificação em escores diferentes para cada sinal e a avaliação em conjunto de todos os achados ultra-sonográficos, o que aumentaria a sensibilidade sem reduzir a especificidade. Grosso modo, a nosso ver, a amniocentese citogenética pode ser proposta quando se identificam escores iguais a 2 ou maiores, levando-se em consideração a relação custo/benefício em face do risco de abortamento provocado pelo exame.

\section{SUMMARY}

Purpose: to calculate sensitivity, specificity and positive and negative predictive values for multiparameter ultrasound scores for Down's syndrome.

Patients and Methods: sensitivity and specificity for Down syndrome were calculated for ultrasound scores in a prospective study of ultrasound signs from 16 to 24 weeks in a high-risk population who denied invasive procedures after genetic counselling. The signs and scores were: femur/foot length $<0,9$ (1), nuchal fold $>5 \mathrm{~mm}(2)$, pyelocaliceal diameter $\geq 5 \mathrm{~mm}(1)$, nasal bones < $6 \mathrm{~mm}$ (1), absent or hypoplastic fifth median phalanx (1) and major structural malformations (2). Complete follow-up was obtained in each case. Genetic amniocentesis was proposed in the case of score 2 or more.

Results: a total of 963 patients were examined from October 93 to December 97 at a mean gestational age of 19.6 (range $16-24$ ) weeks. Women's age ranged from 35 to 47 years (mean 38.8 ) and 18 Down syndrome cases were observed (1.8\%). Sensitivity was $94.5 \%$ (17/18) for score 1 and $73 \%$ (13/18) for score 2 (false positive rate of $9.8 \%$ for score 1 and $4.1 \%$ for score 2). Individual sign sensitivity and specificity were: femur/foot $=16.7 \%$ (3/18) and 96.8\% (915/945); nasal bones $=22.2 \%(4 / 18)$ and $92.1 \%$ (870/945); nuchal fold $=44.4 \%$ $(8 / 18)$ and $96.5 \%$ (912/945); pyelic diameter $=38.9 \%(7 / 18)$ and $94.3 \%$ (891/945); absent phalanx $=22.2 \%(4 / 18)$ and $98.5 \%(912 / 945) ;$ malformation $=22.2 \%(4 / 18)$ and $98.2 \%$ (928/945).

Conclusions: the overall sensitivity for score 1 was high but false positive rates were also high. For score 2, sensibility was still good (73\%) and false positive rate was acceptable (4.1\%). Positive and negative predictive values can be calculated for each prevalence (women's age). More cases are needed to reach final conclusions about this screening method (specially in a low-risk population) although this system has been useful for high-risk patients who deny invasive procedures.

KEY WORDS: Down syndrome. Ultrasound. Prenatal diagnosis. Maternal age. Amniocentesis.

\section{Referências}

1. Allen G, Barof GS. Mongoloid twins and their siblings. Acta Genet 1955; 5: 295.

2. Benacerraf BR. The second-trimester fetus with Down syndrome: detection using sonographic features. Ultrasound Obstet Gynecol 1996; 7: 147-55.

3. Bilardo C. Second trimester ultrasound markers for fetal aneuploidy. Early Hum Dev 1996, 47: (suppl): s31-3.

4. Bromley B, Lieberman E, Benacerraf BR. The incorporation of maternal age into the sonographic scoring index for the detection at 14 20 weeks of fetuses with Down's syndrome. Ultrasound Obstet Gynecol 1997; 10: 321-4.

5. Bunduki V, Margarido PFR. Rastreamento ultrasonográfico de aneuploidias no segundo trimestre. In: Zugaib M, editor. Medicina fetal. 2 ed. São Paulo: Atheneu; 1997. p.590-600.

6. Cuckle H. Biochemical and ultrasound screening for Down'syndrome: rivals or partners? Ultrasound Obstet Gynecol 1996; 7: 236-8.

7. Hahnemann JM, Vejerslev LO. Accuracy of cytogenetic findings on chorionic villus sampling (CVS) diagnostic consequences of CVS mosaicism and non-mosaic discrepancy in centres contributing to EUCROMIC 1986-1992. Prenat Diagn 1997; 17: 801-20.

8. Knight GJ, Palomaki GE, Neveux LM, Fodor KK, Haddow JE. hCG and free beta-subunit as screening tests for Down syndrome. Prenat Diagn 1998; 18: 235-45. 
9. Macintosh M, Ellis A, Cuckle H, Seth J. Variation in biochemical screening for Down's syndrome in the United Kingdom. Br J Obstet Gynaecol 1998; 105: 465-7.

10.Nicolaides KH, Brizot ML, Snijders RJ. Fetal nuchal translucency: ultrasound screening for fetal trisomy in the first trimester of pregnancy. Br J Obstet Gynaecol 1994; 101: 782-6.

11.Snijders RJ, Sebire NJ, Nicolaides KH. Maternal age and gestational age-specific risk for chromossomal defects. Fetal Diagn Ther 1995; 10: 356-67.
12.Vintzileos AM, Campbell WA, Rodis JF, Guzman ER, Smulian JC, Knuppel RA. The use of secondtrimester genetic sonogram in guiding clinical management of patients at increased risk for fetal trisomy 21. Obstet Gynecol 1996; 87: 948-52.

13.Wald NJ, Kennard A, Hachshaw A, McGuirre A. Antenatal screening for Down's syndrome. J Med Screen 1997; 4: 181-246.

14.Wald NJ, Watt HC, Haddow JE, Knight GJ. Screening for Down syndrome at 14 weeks of pregnancy. Prenat Diagn 1998; 18: 291-3. 